Modelling, Analysis and Simulation

Mas On stabilized integration for time-dependent PDEs

B.P. Sommeijer, J.G. Verwer

RePORt MAS-E06 16 JuLY 2006 
Centrum voor Wiskunde en Informatica (CWI) is the national research institute for Mathematics and Computer Science. It is sponsored by the Netherlands Organisation for Scientific Research (NWO).

CWI is a founding member of ERCIM, the European Research Consortium for Informatics and Mathematics.

CWI's research has a theme-oriented structure and is grouped into four clusters. Listed below are the names of the clusters and in parentheses their acronyms.

Probability, Networks and Algorithms (PNA)

Software Engineering (SEN)

\section{Modelling, Analysis and Simulation (MAS)}

Information Systems (INS)

Copyright (C) 2006, Stichting Centrum voor Wiskunde en Informatica

P.O. Box 94079, 1090 GB Amsterdam (NL)

Kruislaan 413, 1098 SJ Amsterdam (NL)

Telephone +31205929333

Telefax +31205924199

ISSN 1386-3703 


\title{
On stabilized integration for time-dependent PDEs
}

\begin{abstract}
An integration method is discussed which has been designed totreat parabolic and hyperbolic terms explicitly and stiff reaction terms implicitly. The method is a special two-step form of the one-step IMEX (Implicit-Explicit) RKC (Runge-Kutta-Chebyshev) method. The special two-step form is introduced with the aim of getting a non-zero imaginary stability boundary which is zero for the one-step method. Having a non-zero imaginary stability boundary allows, for example, the integration of pure advection equations space-discretized with centered schemes, the integration of damped or viscous wave equations, the integration of coupled sound and heat flow equations, etc. For our class of methods it also simplifies the choice of temporal step sizes satisfying the Von Neumann stability criterion, by embedding a thin long rectangle inside the stability region. Embedding rectangles or other tractable domains with this purpose is an idea of Wesseling.
\end{abstract}

2000 Mathematics Subject Classification: 65M12, 65M20, 65L05

1998 ACM Computing Classification System: G.1.1, G.1.7, G.1.8

Keywords and Phrases: Numerical Integration, Stabilized 



\title{
On Stabilized Integration for Time-Dependent PDEs
}

\author{
B.P. Sommeijer and J.G. Verwer \\ $C W I$ \\ P.O. Box 94079, 1090 GB Amsterdam, The Netherlands \\ ben.sommeijer@cwi.nl, jan.verwer@cwi.nl
}

May 30, 2006

\begin{abstract}
An integration method is discussed which has been designed to treat parabolic and hyperbolic terms explicitly and stiff reaction terms implicitly. The method is a special twostep form of the one-step IMEX (Implicit-Explicit) RKC (Runge-Kutta-Chebyshev) method. The special two-step form is introduced with the aim of getting a non-zero imaginary stability boundary which is zero for the one-step method. Having a non-zero imaginary stability boundary allows, for example, the integration of pure advection equations space-discretized with centered schemes, the integration of damped or viscous wave equations, the integration of coupled sound and heat flow equations, etc. For our class of methods it also simplifies the choice of temporal step sizes satisfying the Von Neumann stability criterion, by embedding a thin long rectangle inside the stability region. Embedding rectangles or other tractable domains with this purpose is an idea of Wesseling.
\end{abstract}

2000 Mathematics Subject Classification: Primary: 65M12, 65M20.

1998 ACM Computing Classification System: G.1.1, G.1.7 and G.1.8.

Keywords and Phrases: Numerical Integration, Stabilized Explicit Integration, RungeKutta-Chebyshev Methods, Reactive Flow Problems, Damped Wave Equations, Coupled Sound and Heat Flow.

Note: Work carried out within theme MAS1.

\section{Introduction}

Time-dependent PDE problems of mixed type containing parabolic, hyperbolic and reaction terms typically exhibit wide ranges of spatial and temporal scales, challenging the spatial discretization to avoid fine space grids over the whole domain through adaptivity, and challenging the time integration to avoid an expensive fully implicit treatment of the whole coupled semi-discrete system. For example, detailed chemistry in reactive flows will mostly require an implicit treatment due to stiffness, but if the flow is advection dominated a fully implicit time integration (of all coupled problem terms) is often a great waste and problematical in higher space dimension [9]. Following the method of lines approach, in this paper 
we focus on the efficient time integration of semi-discrete systems composed of coupled terms of mixed type.

We present a modification of the IMplicit-EXplicit (IMEX) Runge-Kutta-Chebyshev (RKC) method in the form of a simple two-step method. Explicit RKC is a second-order, stabilized Runge-Kutta method the stabilization of which is based on the classical three-term Chebyshev recursion. While explicit RKC was originally designed for pure diffusion problems in [4], and for this purpose further discussed and developed in $[12,14,13]$, we have recently modified it towards an IMEX form for incorporating highly stiff reaction terms [11, 15]. This form thus treats diffusion terms explicitly and reaction terms implicitly. Subsequently, by adding more of a certain damping in the scheme, in [16] we have shown that treatment of advection terms is also possible without giving up the essential stabilization properties for diffusion. The result in [16] is a method which treats advection and diffusion terms explicitly and stiff reaction terms implicitly, which is attractive in higher space dimension. This method also enables a high degree of parallelism which is important for truly large scale flow problems.

The stability region of the explicit one-step method (and its IMEX counterpart) is stretched along the negative real axis. Near the origin its boundary comes close to the imaginary axis allowing the integration of slightly dissipative problems. However, since there is no intersection with the imaginary axis, problems having purely imaginary eigenvalues will impede very small temporal step sizes eventually resulting in instability. The special two-step form proposed here has been chosen to overcome this and to provide a non-zero imaginary stability boundary (see Fig. 3.1 for stability region plots). This allows for example the integration of pure advection equations discretized in space with centered schemes, but also problems of a different origin like damped or viscous wave equations [3, 8], coupled sound and heat flow problems [10], or the Maxwell equations coupled with nonlinear heat equations [19]. An additional advantage is that it also simplifies the choice of temporal step sizes satisfying the Von Neumann stability criterion by embedding a thin and very long rectangle inside the stability 'region, as illustrated in Fig. 3.1. Embedding rectangles or other tractable domains with this purpose is an idea of Wesseling $[17,18]$.

In Section 2 we will briefly review the explicit one-step method and its IMEX form. The two-step form with its stability regions is derived in Section 3. Section 4 is devoted to three example problems so as to illustrate the applicability of stabilized integration. Here we treat a damped wave equation, a system for coupled sound and heat flow, and a stiff advection-diffusion-reaction system. Section 5 concludes the paper with some final remarks and some recent related literature.

\section{The one-step method}

Historically the principal goal when constructing Runge-Kutta methods was to achieve the highest order possible with a given number of stages $s$. Stabilized methods like RKC are different in that usually a low order is chosen whereas additional stages are exploited to increase the region of absolute stability. Originally the RKC method was intended for semidiscrete parabolic PDE problems. Correspondingly, the original method is stable on a strip containing a long segment of the negative real axis. The wider the strip, the greater the applicability of the method, but the most important characteristic of the formula is the length of the segment, the real stability boundary, which increases quadratically with $s$. For 
ODE initial value problems $w^{\prime}=F(t, w)$ the explicit one-step method reads

$$
\begin{aligned}
W_{0} & =w_{n}, \\
W_{1} & =W_{0}+\tilde{\mu}_{1} \tau F_{0}, \\
W_{j} & =\left(1-\mu_{j}-\nu_{j}\right) W_{0}+\mu_{j} W_{j-1}+\nu_{j} W_{j-2}+\tilde{\mu}_{j} \tau F_{j-1}+\tilde{\gamma}_{j} \tau F_{0}, \\
w_{n+1} & =W_{s}, \quad j=2, \ldots, s,
\end{aligned}
$$

where $w_{n}, w_{n+1}$ denote the sought approximations at times $t_{n}, t_{n+1}$ and $\tau=t_{n+1}-t_{n}$ is the step size which may vary in time. Although this formula is not in the canonical Runge-Kutta form, it belongs to the class of $s$-stage explicit Runge-Kutta methods. The $W_{j}$ are internal vectors approximating $w\left(t_{n}+c_{j} \tau\right)$ and $F_{j}$ denotes $F\left(t_{n}+c_{j} \tau, W_{j}\right)$ with $0 \leq c_{j} \leq 1$. Note the recursive form of the $W_{j}$ by which only 5 arrays of storage are needed independent of the value for $s$. All coefficients are available in analytical form for arbitrary $s \geq 2$, see $[13,15]$ and earlier references and [12] for a Fortran 77 code.

When applied to the scalar stability test equation $w^{\prime}=\lambda w, \lambda \in \mathbb{C}$ (having Fourier-von Neumann analysis in mind), the second-order consistent stability function

$$
P_{s}(z)=1-b_{s} T_{s}\left(\omega_{0}\right)+b_{s} T_{s}\left(\omega_{0}+\omega_{1} z\right)
$$

is obtained, where $T_{s}$ is the first kind Chebyshev polynomial of degree $s$ and

$$
\omega_{0}=1+\varepsilon / s^{2}, \quad \omega_{1}=T_{s}^{\prime}\left(\omega_{0}\right) / T_{s}^{\prime \prime}\left(\omega_{0}\right), \quad b_{s}=T_{s}^{\prime \prime}\left(\omega_{0}\right) /\left(T_{s}^{\prime}\left(\omega_{0}\right)\right)^{2} .
$$

The parameter $\varepsilon \geq 0$ is still free and is available for damping. If $\varepsilon>0,0<P_{s}(z)<1$ in the interior of the real stability interval $[-\beta(s), 0]$. With $\varepsilon \approx 0$ we have $\beta(s) \approx 0.66\left(s^{2}-1\right)$. This choice is appropriate for pure diffusion problems. For advection-diffusion problems $\varepsilon$ should be chosen larger to get wider stability regions and, at the origin, their boundaries closer to the imaginary axis. In [16] the value $\varepsilon=10$ has been used. Then upwind discretizations yield appropriate CFL numbers at the expense of a reduction in $\beta(s)$ to $\approx 0.34\left(s^{2}-1\right)$. Note that for $s=2$ method (2.1) reduces to the explicit trapezoidal rule which for the third-order upwind-biased advection scheme results in a critical CFL number of 0.87 [5]. For increasing $s$ this critical CFL number monotonically increases to 1.7. However, for all $\varepsilon \geq 0$ the intersection of the stability region with the imaginary axis is zero.

The IMEX counterpart of (2.1) is defined for ODE systems [15, 16],

$$
w^{\prime}=F(t, w), \quad F(t, w)=F_{E}(t, w)+F_{I}(t, w)
$$

with $F_{E}, F_{I}$ representing terms that will be treated Explicitly and Implicitly:

$$
\begin{aligned}
W_{0}= & w_{n}, \\
W_{1}= & W_{0}+\tilde{\mu}_{1} \tau F_{E, 0}+\tilde{\mu}_{1} \tau F_{I, 1}, \\
W_{j}= & \left(1-\mu_{j}-\nu_{j}\right) W_{0}+\mu_{j} W_{j-1}+\nu_{j} W_{j-2}+\tilde{\mu}_{j} \tau F_{E, j-1}+\tilde{\gamma}_{j} \tau F_{E, 0}+ \\
& {\left[\tilde{\gamma}_{j}-\left(1-\mu_{j}-\nu_{j}\right) \tilde{\mu}_{1}\right] \tau F_{I, 0}-\nu_{j} \tilde{\mu}_{1} \tau F_{I, j-2}+\tilde{\mu}_{1} \tau F_{I, j}, } \\
w_{n+1}= & W_{s}, \quad j=2, \ldots, s .
\end{aligned}
$$

Here $F_{E, j}=F_{E}\left(t_{n}+c_{j} \tau, W_{j}\right)$ and $F_{I, j}=F_{I}\left(t_{n}+c_{j} \tau, W_{j}\right)$. For zero $F_{I}$ the scheme is identical to $(2.1)$. One can see that for non-zero $F_{I}$ this term is treated implicitly in a backward Euler 
way at any $j$-th stage, using the same coefficient $\tilde{\mu}_{1}$. Of importance is that the stationary state equation $F_{E}(t, w)+F_{I}(t, w)=0$ is respected [15] and that we do not split $F_{E}$ and $F_{I}$ as in time (operator) splitting. If the implicit term does not involve coupling over the space grid, the implicit computation becomes relatively cheap (certainly with parallelization).

When applied to the scalar stability test equation $w^{\prime}=\lambda_{E} w+\lambda_{I} w$, we get the counterpart of $(2.2)$,

$$
R_{s}\left(z_{E}, z_{I}\right)=1-b_{s} T_{s}\left(\omega_{0}\right)+b_{s} T_{s}\left(\frac{\omega_{0}+\omega_{1} z_{E}}{1-\frac{\omega_{1}}{\omega_{0}} z_{I}}\right),
$$

where $z_{E}=\tau \lambda_{E}, z_{I}=\tau \lambda_{I}$. From this expression, linear stability (in the sense of Fouriervon Neumann) can be concluded for all $\left(z_{E}, z_{I}\right)$ such that $z_{I} \leq 0$ and $z_{E}$ belongs to a convex region inside the stability region of $P_{s}(z)$ (e.g. ellipses or ovals as in [16]). Hence the IMEX form provides unconditional stability for real negative implicit eigenvalues, such as real negative reaction eigenvalues, and maintains the stability for explicit eigenvalues, such as transport eigenvalues in an advection-diffusion? context. The order of consistency of (2.5) formally drops down to one, but with a very small error coefficient for the implicitly treated part if $s$ gets large. See [15] for further details.

\section{The two-step method}

Suppose $w_{n-1}, w_{n}$ have been computed by the two-step method at times $t_{n-1}, t_{n}=t_{n-1}+$ $\tau_{n-1}$. Using the variable step size $\tau_{n}=t_{n+1}-t_{n}$, the two-step method then defines the approximation $w_{n+1}$ at the forward time level $t_{n+1}$ by

$$
w_{n+1}=\alpha_{-1} w_{n-1}+\alpha_{0} w_{n}+\alpha_{\eta} \tilde{w}_{n+\eta},
$$

where $\tilde{w}_{n+\eta}$ is the result of the one-step method (2.1) or (2.5) taken from $w_{n}$ with a step size $\eta \tau_{n}$. Hence we first apply the one-step method at $t=t_{n}$ as above, but with a step size $\eta \tau_{n}$, and then form the new forward two-step result $w_{n+1}$ by means of (3.1). Implementing this two-step method is trivial if the one-step method has been coded, see [12] for a Fortran 77 code of (2.1) and [11] for a Fortran 90 code of (2.5). To obtain the extra starting value, the first step can be done with the one-step method.

\subsection{Consistency conditions}

The new coefficients $\alpha_{-1}, \alpha_{0}, \alpha_{\eta}, \eta$ will be first restricted to give second-order consistency for nonlinear systems $w^{\prime}=F(t, w)$ assuming that $\tilde{w}_{n+\eta}$ has been obtained with the secondorder explicit method (2.1). For this purpose we can take $F(t, w)=A w$ with $A$ constant, for which the one-step method for $s \geq 3$ satisfies the expansion

$$
\tilde{w}_{n+\eta}=w+\eta \tau_{n} w^{\prime}+\frac{1}{2} \eta^{2} \tau_{n}^{2} w^{\prime \prime}+\frac{1}{6} c \eta^{3} \tau_{n}^{3} w^{\prime \prime \prime}+\mathcal{O}\left(\tau_{n}^{4}\right), \quad c \neq 0
$$

where $w=w\left(t_{n}\right)$. The constant $c$ is found by expanding $P_{s}(z)$ given in $(2.2)$ at $z=0$,

$$
P_{s}(z)=1+z+\frac{1}{2} z^{2}+\frac{1}{6} c z^{3}+\cdots, \quad c=\frac{T_{s}^{\prime}\left(\omega_{0}\right) T_{s}^{\prime \prime \prime}\left(\omega_{0}\right)}{T_{s}^{\prime \prime}\left(\omega_{0}\right)^{2}} .
$$


Denoting with $r_{n}=\tau_{n} / \tau_{n-1}$ the step size ratio, we then find for second-order consistency for general nonlinear systems the conditions

$$
\alpha_{0}=1-\alpha_{-1}-\alpha_{\eta}, \quad \alpha_{\eta}=\frac{1+r_{n}}{\eta\left(1+\eta r_{n}\right)}, \quad \alpha_{-1}=\frac{r_{n}^{2}(1-\eta)}{1+\eta r_{n}},
$$

where $\eta$ still free. It is possible to impose third-order consistency for the linear problem $w^{\prime}=A w$ by defining $\eta$ through $\left(c r_{n}\right) \eta^{2}+\left(1-r_{n}\right) \eta-1=0$. There are always two real roots for $\eta$, one negative and one positive. We need of course the positive root. For example, for constant step sizes we then have $\eta=1 / \sqrt{c}$.

For the IMEX method (2.5) the order again drops down to one, but with a very small error constant for the implicitly treated part for $s$ large. Observe that all coefficients depend on $c$, hence on $\omega_{0}$, hence on $s$ and $\varepsilon$ through (2.3). This dependence is weak and decreases rapidly with $s$ and in all cases moderate values are found. For example, for $s=3$ we have $c=\left(4 \omega_{0}^{2}-1\right) / 8 \omega_{0}^{2}=1 / 2-1 /\left(8(1+\varepsilon / 9)^{2}\right)$, revealing a weak dependence on $\varepsilon$.

It should be noted that always $1<\eta<2$. For problems not defined beyond an output time $t=T$, having $\eta>1$ implies that the final integration step should be carried out by means of the one-step formula, similar as the initial step. Finally, $s \geq 3$ is required since the requirement $c \neq 0$ implies $s \geq 3$ for (2.1). A consequence is that the $s=2$ formula of (2.1), being the explicit trapezoidal rule, cannot be extended to (linear) order three within the format (3.1).

\subsection{Stability regions}

Our aim is to provide (3.1) with a non-zero imaginary stability boundary. For that purpose we consider linear stability for constant step sizes, assume the explicit method (2.1) for providing $\tilde{w}_{n+\eta}$, and let $\eta=1 / \sqrt{c}$ giving third-order consistency for linear problems.

Consider the corresponding characteristic equation

$$
\zeta^{2}-\left(\alpha_{0}+\alpha_{\eta} P_{s}(\eta z)\right) \zeta-\alpha_{-1}=0,
$$

and let $\beta_{R e}(s)$ and $\beta_{I m}(s)$ denote the real and imaginary stability boundary. Imposing the root condition [5] defines the stability region, $\beta_{R e}(s)$ and $\beta_{I m}(s)$, and assures zero-stability (for $z=0$ ). The stability regions are constructed numerically from the root condition. Herewith the damping parameter $\varepsilon$ can be used to adjust the shape of the regions. Taking $\varepsilon \gg 1$ will reduce $\beta_{R e}(s)$ considerably. On the other hand, the dependence of $\beta_{I m}(s)$ on $\varepsilon$ turns out to be minor. In connection with $\beta_{I m}(s)$ the case $s=3$ appears to be special. We have $\beta_{\operatorname{Im}}(3)=\sqrt{3}$ for all $\varepsilon \geq 0$ which also happens to be the imaginary stability boundary of any one-step three-stage, third-order explicit Runge-Kutta method. The independence of $\beta_{I m}(3)$ on $\varepsilon$ is due to the fact that precisely at $z=i \sqrt{3}$ the modulus of the largest root of (3.3) is independent of $\varepsilon$ and equal to 1 . For increasing $s$ the boundary $\beta_{I m}(s)$ does slightly increase, and somewhat stronger for larger $\varepsilon$. Illustrations of stability regions are given in Fig. 3.1. Their defining $\varepsilon$-values are found in Table 3.1.

Would we refrain from third-order consistency for linear problems, the coefficient $\eta$ is free to maximize $\beta_{I m}(s)$. A numerical search has shown that for $s=3$ the imaginary boundary can be increased to 2.0 with a similar increase for $s>3$. This however comes at the expense of significantly smaller real stability boundaries so that we prefer to use the coefficients giving third-order consistency for linear problems. 

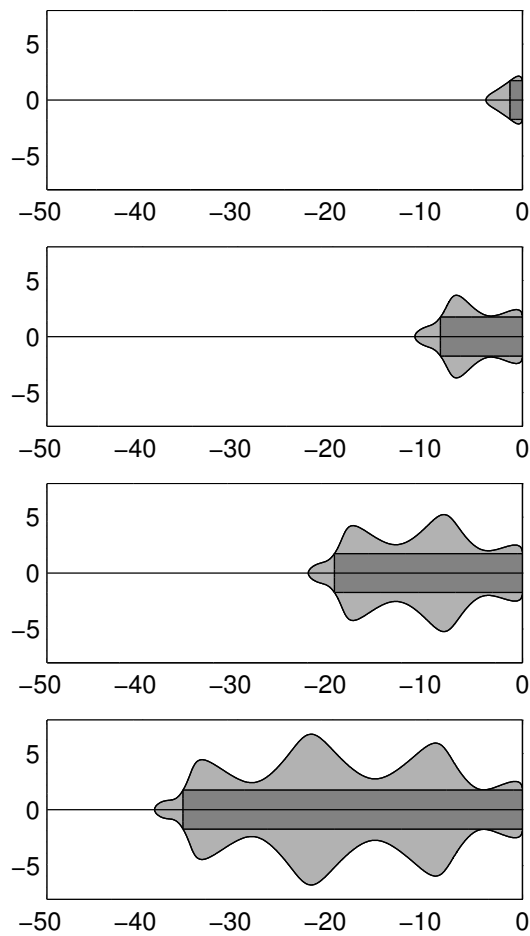
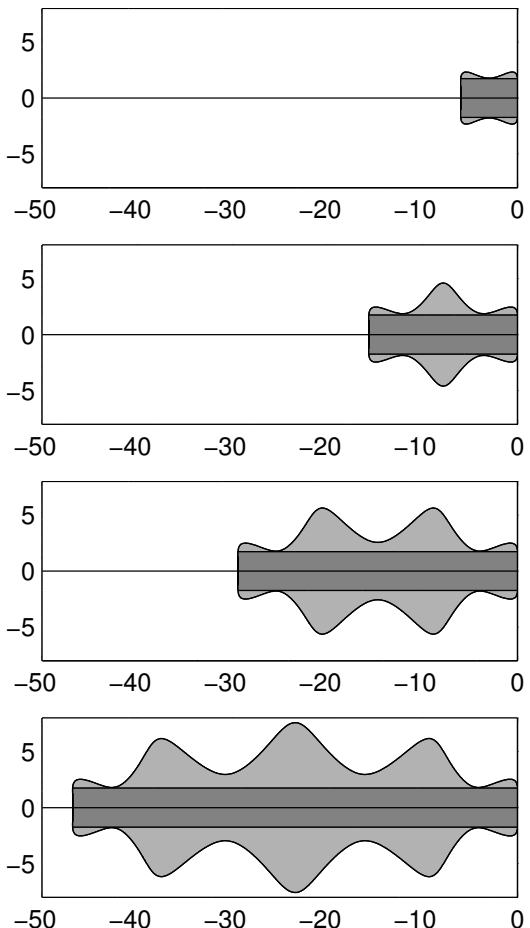

Figure 3.1: Stability regions with embedded rectangles. Left for $s=3,5,7,9$. Right for $s=4,6,8,10$.

\begin{tabular}{c||c|c|c|c|c|c|c|c|c}
$s$ & 3 & 4 & 5 & 6 & 7 & 8 & 9 & 10 & $>10$ \\
\hline$\varepsilon$ & $\infty$ & 4 & 2 & 1.5 & 1.5 & 1.0 & 0.9 & 0.9 & 0.9 \\
$S_{R e}(s) / s^{2}$ & 0.14 & 0.37 & 0.34 & 0.43 & 0.40 & 0.45 & 0.44 & 0.45 & 0.45 \\
\hline
\end{tabular}

Table 3.1: Values for $\varepsilon$ and $S_{R e}(s)$ rounded to two decimal places for the rectangles from Fig. 3.1.

\subsection{Embedded thin rectangles}

To facilitate the selection of $\tau$ according to Fourier-von Neumann stability (for example for the most common advection-diffusion discretizations), we will now embed rectangles

$$
\mathcal{R}(s)=\left\{z \in \mathbb{C}: z=x+i y,-S_{R e}(s) \leq x \leq 0,|y| \leq S_{\text {Im }}(s)\right\}
$$

within the stability regions, such that $S_{R e}(s) \approx \beta_{R e}(s)$ and $S_{I m}(s) \approx \beta_{I m}(s)$. These rectangles are then used for the step size selection, an idea proposed in Wesseling [17], see also Section 5.7 in [18] and [16] where other embedded domains are considered.

We choose $\sqrt{3}$ for $S_{I m}(s)$ for all $s \geq 3$, and adjust $\varepsilon$ to get $S_{R e}(s)$ as large as possible. This approach is convenient. Because given a semi-discrete PDE problem with purely imaginary eigenvalues, or eigenvalues close to the imaginary axis, we then can determine $\tau$ from the condition that the imaginary part of any occurring $z$-value will lie in a rectangle and hence in a stability region. Having dealt with the imaginary parts and thus have a selected $\tau$ at hand, we subsequently can choose the smallest value for $s$ such that the real part of any occurring $z$-value will lie in the rectangle. At this point we thus exploit the stabilization for diffusion terms by which we can avoid a diffusion step size restriction. After some trial and 
error computations, we have concluded that this embedded rectangle approach works best for small $s$ if $\varepsilon$ is relatively large, which is e.g. advantageous for pure advection problems. On the other hand, for large $s$ smaller $\varepsilon$-values can be taken which is advantageous for pure diffusion problems.

The results are given in Fig. 3.1 and Table 3.1. The figure shows embedded rectangles for $s=3, \ldots, 10$, while the table gives the corresponding values for $\varepsilon$ and $S_{R e}(s)$. Observe that the most right column in the table applies to arbitrary $s>10$ and recall that $S_{I m}(s)=\sqrt{3}$ for all $s \geq 3$. Due to symmetry we have $S_{R e}(s)=\beta_{R e}(s)$ for $s$ even while for $s$ large $\beta_{R e}(s) \approx 0.45 s^{2}$. This result is quite satisfactory in view of the fact that the optimal real stability boundary for third-order stabilized polynomials (for one-step methods) is close to $0.49 s^{2}$, see Sect. V.1.1 in [5]. Note that for $s=3$ the optimal value for $\varepsilon$ for embedding the rectangle is infinity. This results in a special formula for the one-step method (formula (3.3) in $[16])$.

Finally assume that the IMEX method (2.5) is used to obtain the one-step result $\tilde{w}_{n+\eta}$. In the characteristic equation (3.3) we then have to replace $P_{s}(\eta z)$ by $R_{s}\left(\eta z_{E}, \eta z_{I}\right)$ defined in (2.6). By rewriting $R_{s}\left(\eta z_{E}, \eta z_{I}\right)$ to

$$
P_{s}(\eta \tilde{z})=1-b_{s} T_{s}\left(\omega_{0}\right)+b_{s} T_{s}\left(\omega_{0}+\omega_{1} \eta \tilde{z}\right), \quad \tilde{z}=\frac{z_{E}+z_{I}}{1-\frac{\omega_{1}}{\omega_{0}} \eta z_{I}}
$$

it follows that for stability we can again consider (3.3) with $z$ replaced by $\tilde{z}$. It thus can be shown that if $z_{E}$ lies in a rectangle, $\tilde{z}$ also lies in this rectangle for any $z_{I} \leq 0$, assuring linear stability.

\subsection{Fourier-von Neumann stability for advection-diffusion}

Using the embedded rectangle approach we can easily give Fourier-von Neumann stability restrictions on $\tau$ and $s$ for standard advection-diffusion discretizations of the test model

$$
u_{t}+\sum_{k} a_{k} u_{x_{k}}=\sum_{k} d_{k} u_{x_{k} x_{k}}+r u .
$$

The reaction term $r u$ has been added to emphasize that all results are valid for the IMEX two-step scheme for any real non-positive reaction eigenvalue $r$. Because the IMEX scheme is unconditionally stable for these eigenvalues, $r$ will not appear in the stability restrictions.

Let us suppose spatial discretization on a direction-wise uniform Cartesian grid with grid sizes $h_{k}$ by means of the following schemes [5]: for advection 1-st order upwind, 2-nd order central, 3-rd order upwind-biased, 4-th order central, and for diffusion 2-nd order central and 4 -th order central. Denote

$$
A=\sum_{k} \frac{\left|a_{k}\right|}{h_{k}}, \quad D=\sum_{k} \frac{d_{k}}{h_{k}^{2}} .
$$

The step size restriction on the imaginary parts of the eigenvalues emerging from Fourier-von Neumann analysis then becomes the CFL condition

$$
\tau \leq\left\{\begin{aligned}
\sqrt{3} / A & : \text { 1-st and 2-nd order advection discretization } \\
\frac{5}{7} \sqrt{3} / A & : \text { 3-rd and 4-th order advection discretization }
\end{aligned}\right.
$$

The condition on the number of stages $s$, guaranteeing that the real parts of all emerging eigenvalues lie within the rectangle, is $\tau \sigma \leq S_{R}(s)=c_{R e}(s) s^{2}$ where $\sigma$ is in modulus the 
largest real part and $c_{R e}(s)$ the constant given in Table 3.1. For the 2-nd order diffusion discretization the $\sigma$-values are

$$
\sigma=\left\{\begin{aligned}
2 A+4 D & : \text { 1-st order advection discretization } \\
4 A / 3+4 D & : \text { 3-rd order advection discretization } \\
4 D & : \text { 2-nd and 4-th order advection discretization }
\end{aligned}\right.
$$

while for the 4-th order diffusion discretization the term $4 D$ is to be replaced by $16 D / 3$.

We thus should take the smallest number of stages $s$ satisfying $s^{2} \geq \tau \sigma / c_{R e}(s)$. If $D=0$ (pure advection) the smallest value is of course $s=3$. If $A=0$ (pure diffusion) we have no step size restriction. In all other cases with Table 3.1 at hand and $\tau$ and $\sigma$ given, searching a minimal value for $s$ is a trivial task because $c_{R e}(s)$ is constant for $s \geq 10$.

\section{Numerical examples}

\subsection{A damped wave equation}

As a first test problem we consider the scalar damped wave equation (see $[3,8]$ )

$$
\gamma u_{t}+u_{t t}=\nabla \cdot\left(D \nabla u+Q \nabla u_{t}\right)+S
$$

where $\gamma \geq 0, D$ and $Q$ are symmetric nonnegative diffusion tensors and $S$ is a source, all possibly dependent on space, time and $u$. This equation represents an interesting class of problems containing the classic two-way wave equation having a purely imaginary spectrum and strongly damped equations having a real negative spectrum. It thus is of clear interest for a numerical study of the two-step explicit RKC method. Here we confine ourselves to the $2 \mathrm{D}$ problem

$$
\gamma u_{t}+u_{t t}=\left(d_{1} u_{x}+q_{1} u_{t x}\right)_{x}+\left(d_{2} u_{y}+q_{2} u_{t y}\right)_{y}+S,
$$

defined on the unit square, with given initial conditions for $u$ and $u_{t}$ at $t=0$, and zero-flux boundary conditions for $t \geq 0$. We emphasize that the restriction to $2 \mathrm{D}$ is not essential and merely made for the convenience of obtaining and presenting numerical illustrations. The explicit nature of the integrator does allow treatment of 3D problems extended with the $z$ direction in the same manner as we will use the $2 \mathrm{D}$ problem here. For numerical integration we write (4.2) as

$$
\begin{aligned}
& u_{t}=v, \\
& v_{t}=-\gamma v+\left(d_{1} u_{x}+q_{1} v_{x}\right)_{x}+\left(d_{2} u_{y}+q_{2} v_{y}\right)_{y}+S,
\end{aligned}
$$

and for spatial discretization we use second-order central differences on a uniform cellcentered $N \times N$ grid of width $h=1 / N$. This grid is convenient to implement zero fluxes at boundaries.

Assuming constant coefficients and periodicity, with this grid and this spatial discretization, Fourier-von Neumann time-stepping stability requires $z_{ \pm}=\tau \lambda_{ \pm}$to lie inside the stability region, where $\tau$ is the step size,

$$
\begin{aligned}
& \lambda_{ \pm}=-\frac{1}{2}\left(\alpha_{q}+\gamma\right) \pm \frac{1}{2} \sqrt{\left(\alpha_{q}+\gamma\right)^{2}-4 \alpha_{d}} \\
& \alpha_{d}=\frac{4 d_{1}}{h^{2}} \sin ^{2}\left(\xi_{1}\right)+\frac{4 d_{2}}{h^{2}} \sin ^{2}\left(\xi_{2}\right), \quad 0 \leq \xi_{1}, \xi_{2} \leq \pi
\end{aligned}
$$


and $\alpha_{q}$ is defined in the same way as $\alpha_{d}\left(q_{1}, q_{2}\right.$ instead of $\left.d_{1}, d_{2}\right)$. If $\gamma=q_{1}=q_{2}=0$ (no damping) all eigenvalues are purely imaginary with a maximum modulus of $2 h^{-1} \sqrt{d_{1}+d_{2}}$. In this undamped case two-step RKC can be used with $s=3$ and the imaginary stability boundary $\beta_{I m}=\sqrt{3}$ then results in the time step restriction

$$
\tau \leq \frac{h \sqrt{3}}{2 \sqrt{d_{1}+d_{2}}},
$$

which is also used for $s>3$. With damping, all eigenvalues do have a negative real part which is largest for $\lambda_{-}$which in turn is bounded from below by $-\left(\alpha_{q}+\gamma\right)$. Using this safe bound and the given restriction on the step size $\tau$, the minimal required number of stages $s$ is then easily derived from Table 3.1 through

$$
\tau\left(\frac{4}{h^{2}}\left(q_{1}+q_{2}\right)+\gamma\right) \leq c_{R e}(s) s^{2}
$$

If both (4.5) and (4.6) are satisfied all values $z_{ \pm}$lie inside the stability rectangle of the method guaranteeing Fourier-von Neumann stability. Of course, for the damped case it might pay to compute the maximal imaginary and negative real parts more accurately so as to integrate with a larger $\tau$ and/or a smaller number of stages $s$.

Example. Using a constant step size $\tau$, integrations have been carried out with the explicit two-step method for a zero initial function for $u$ and $u_{t}$, constant problem coefficients $\gamma=0, d_{1}=0.01, d_{2}=1$, spatially dependent coefficients $q_{1}=q_{2}=q$ and a spatially dependent source function $S$ :

$$
\begin{aligned}
& q(x, y)=0.1 e^{-100\left((x-0.25)^{2}+(y-0.25)^{2}\right)}, \\
& S(x, y)=100 e^{-500\left((x-0.75)^{2}+(y-1)^{2}\right)}+100 e^{-500\left((x-0.25)^{2}+(y-1)^{2}\right)} .
\end{aligned}
$$

The localization of $S$ implies that two waves are excited, one at the boundary point $(x, y)=$ $\left(\frac{3}{4}, 1\right)$ and one at $\left(\frac{1}{4}, 1\right)$. By the choice of $d_{1}, d_{2}$ both waves travel to the opposite $y=0$ boundary, the first undamped and the second damped near the location $\left(\frac{1}{4}, \frac{1}{4}\right)$ of the damping function $q$. Wave profiles nicely revealing the smoothing effect of the damping are shown in Figure 4.1. We remark that the chosen function and coefficient values address no specific physical situation; they merely serve to illustrate the numerics.

Because the peaked damping function $q$ given in (4.7) is very close to zero in part of the domain, with this setup we encounter, after linearization, eigenvalues $\lambda_{ \pm}$very close to the imaginary axis as well as eigenvalues with a large negative real part which gives a type of spectrum the explicit two-step method can deal with, as opposed to the one-step method which will become unstable. The semi-discretization has been carried out on coarse and fine grids, using $N=50(\times 2) 400$. Because the semi-discrete system $w^{\prime}=F(t, w)$ is linear with constant coefficients in time, the two-step method integrates with third-order consistency. Hence we expect that the spatial errors dominate, being of second order. The step size $\tau$ was defined by (4.5) giving $\tau \approx 0.86 / N$. With this $\tau$ at hand, the minimal number of required stages $s$ was determined from (4.6) using the maximal value of the damping function $q$. This gives the inequality $s^{2} \geq 0.69 \mathrm{~N} / c_{R e}(s)$.

The resulting values of $\tau$ and $s$ are given in Table 4.1. Note that the stage number $s$ increases with $\sqrt{N}$ due to the fact that $\tau$ is proportional to $1 / N$. Table 4.1 also gives 

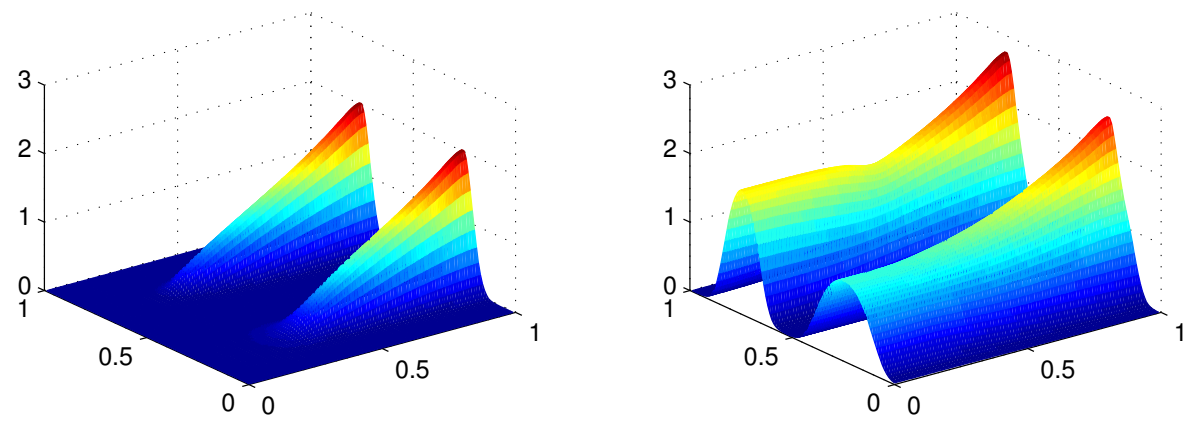

Figure 4.1: Damped wave equation: wave profiles computed on the $100 \times 100$ grid for $t=0.75$ (left) and $t=1.5$ (right).

maximum values for the $u$-approximation taken over the grids at the end time $t=1.5$, so as to provide an accuracy measure. The results are very satisfactory. Two-step RKC solves this problem routinely and accurately and given that the computation is explicit, even on fine grids the used step sizes $\tau$ and numbers of stages $s$ are quite acceptable.

Finally, would we replace the peaked damping function $q$ by one which stays away from zero, the eigenvalues $\lambda_{ \pm}$given by (4.4) would have a strictly negative real part. In such situations also the one-step method could be considered. For example, for $q_{1}=q_{2}=0.1$, the one-step method is stable when using the step sizes and stage numbers of Table 4.1.

\begin{tabular}{l||c|c|c} 
& $\tau$ & $s$ & $u_{\max }$ at $t=1.5$ \\
\hline$N=50$ & 0.0172 & 9 & 2.79447 \\
$N=100$ & 0.0086 & 13 & 2.77026 \\
$N=200$ & 0.0043 & 18 & 2.77600 \\
$N=400$ & 0.0022 & 25 & 2.77743 \\
\hline
\end{tabular}

Table 4.1: Damped wave equation: step sizes $\tau$, numbers of stages $s$ and maxima for $u$.

\subsection{Coupled sound and heat flow}

As a second example we consider the scaled linearized equations for coupled sound and heat flow. Expressing conservation of mass, momentum and energy, these equations read (see e.g. [10], Sect. 10.4)

$$
v_{t}=c \nabla \cdot \mathbf{u}, \quad \mathbf{u}_{t}=c \nabla v-c(\gamma-1) \nabla e, \quad e_{t}=d \Delta e-c \nabla \cdot \mathbf{u} .
$$

The solution variables $v, \mathbf{u}$ and $e$ represent respectively specific volume, material velocity and specific internal energy; $c$ is the isothermal sound speed, $\gamma>1$ the ratio of specific heat, and $d \geq 0$ the thermal conductivity coefficient. We consider this system in $2 \mathrm{D}$ on the unit square assuming periodicity for boundary conditions. For spatial discretization the fourth-order central scheme on a uniform grid of width $h$ will be used and time integration will be done with two-step RKC. 
In Fourier space the 2D PDE system (4.8) gives the 4-component ODE system

$$
\frac{d}{d t}\left(\begin{array}{c}
\xi \\
\eta_{1} \\
\eta_{2} \\
\zeta
\end{array}\right)=\left(\begin{array}{cccc}
0 & c \alpha_{1} & c \alpha_{2} & 0 \\
c \alpha_{1} & 0 & 0 & c(1-\gamma) \alpha_{1} \\
c \alpha_{2} & 0 & 0 & c(1-\gamma) \alpha_{2} \\
0 & -c \alpha_{1} & -c \alpha_{2} & d\left(\delta_{1}+\delta_{2}\right)
\end{array}\right)\left(\begin{array}{l}
\xi \\
\eta_{1} \\
\eta_{2} \\
\zeta
\end{array}\right),
$$

where $\xi$ belongs to $v, \eta_{1}$ and $\eta_{2}$ to the two velocity components in $x$ and $y$ direction, and $\zeta$ to the energy component $e$. The coefficients $\alpha_{1}, \alpha_{2}$ and $\delta_{1}, \delta_{2}$ are eigenvalues of the spatial operators for, respectively, $\partial / \partial x$ and $\partial / \partial y$ and $\partial^{2} / \partial x^{2}$ and $\partial^{2} / \partial y^{2}$. For example, for the fourth-order central scheme, $\alpha_{1}=\frac{i}{3 h} \sin \left(\omega_{1}\right)\left(4-\cos \left(\omega_{1}\right)\right)$ with $0 \leq \omega_{1} \leq 2 \pi$, giving a maximum modulus bounded by $\frac{7}{5} h^{-1}$. Likewise, $\delta_{1}$ is real, non-positive with a maximum modulus of $\frac{16}{3} h^{-2}$, being given by $\delta_{1}=-\frac{1}{3 h^{2}}\left(7-8 \cos \left(\omega_{1}\right)+\cos ^{2}\left(\omega_{1}\right)\right)$. The 4-dimensional system defines the eigenvalues $\lambda$ and the values $z=\tau \lambda$ from which we can derive step size restrictions through the stability rectangles of two-step RKC. Unfortunately, the resulting cubic characteristic equation (we have one zero eigenvalue) is not feasible for elaboration. We therefore proceed with the two much more simple separate cases $c=0$ (diffusion only) and $d=0$ (zero diffusion).

First let $d=0$. Then the characteristic equation reads $\lambda\left(\lambda^{2}-\gamma c^{2}\left(\alpha_{1}^{2}+\alpha_{2}^{2}\right)\right)=0$. Using the maximum modulus of $\alpha_{1}, \alpha_{2}$ and the imaginary stability boundary $\beta_{I m} \geq \sqrt{3}$ of two-step RKC, we get the step size restriction (see (3.7))

$$
\tau \leq \frac{5 \sqrt{3}}{7 \sqrt{2}} \frac{1}{c \sqrt{\gamma}} h .
$$

The number of stages $s$ for two-step RKC can now be set to 3 and the Fourier-von Neumann stability analysis rigorously applies. Next assume zero sound speed, having just the heat equation. We then immediately arrive at a restriction similar to (4.6), that is,

$$
\tau \leq \frac{3}{32} \frac{h^{2}}{d} c_{R e}(s) s^{2} .
$$

As common for a stabilized method for the heat equation, we then have the freedom to first make an appropriate choice for $\tau$ and subsequently we can adjust the number of stages $s$ using Table 3.1 and (4.10). Again Fourier-von Neumann stability analysis rigorously applies.

In the mixed case we are aiming at where simultaneously $d>0$ and $c>0$, we apply Fourier-von Neumann analysis heuristically. That is, we first impose (4.9) prescribing $\tau$. Subsequently, $s$ is chosen by means of (4.10) and Table 3.1. In the numerical tests described below this heuristic strategy has resulted in stable computations.

Example. As time interval we have chosen $0 \leq t \leq T=1$, as initial velocities in $x$ and $y$ direction the periodic functions $u_{1}=\frac{2}{5} \pi \sin ^{2}(\pi x) \sin (2 \pi y)$ and $u_{2}=-\frac{1}{5} \pi \sin ^{2}(\pi y) \sin (2 \pi x)$, and a zero initial field for $v$ and $e$. The problem coefficients are given by $c=1, \gamma=3$ and for $d$ we have chosen the peaked function $d=0.1 \sin ^{10}(\pi x) \sin ^{10}(\pi y)$. With this setup we encounter, after linearization, eigenvalues $\lambda_{ \pm}$very close to the imaginary axis since $d$ is close to zero on part of the domain. This gives, after freezing coefficients, a type of spectrum the explicit two-step method can deal with, as opposed to the one-step method which 
will become unstable. Similar as for the damped wave equation, the choices made are not physically based; they only serve to illustrate the numerics.

Using the above strategy for selecting the step size $\tau$ (taken constant) and number of stages $s$, the coupled sound and heat flow problem was then solved on coarse and fine grids using $N=50(\times 2) 400$. Figure 4.2 shows plots of approximations to $u_{2}$ and $e$ computed at the $100 \times 100$ grid at the end time $t=1$. The used values of $\tau$ and $s$ are given in Table 4.2. Note that like for the damped wave equation, the stage number $s$ increases with $\sqrt{N}$ due to the fact that $\tau$ is proportional to $1 / N$. Table 4.2 also gives maximum values for the approximation to $e$ taken over the grids at the end time $t=1.0$, so as to provide an accuracy measure. Again the results are very satisfactory. Explicit two-step RKC also solves this problem routinely and accurately. Because we use here a fourth-order spatial discretization, the order three of two-step RKC will prevail for decreasing $\tau$ and $h$ (the semi-discrete problem is linear and has constant coefficients). Finally, would we have a strictly positive thermal conductivity coefficient, say $d=0.1$ rather than the chosen peaked function, the one-step method is also stable for the current problem.

\begin{tabular}{l||c|c|c} 
& $\tau$ & $s$ & $e_{\max }$ at $t=1.0$ \\
\hline$N=50$ & 0.0101 & 8 & 0.038500 \\
$N=100$ & 0.0051 & 11 & 0.038624 \\
$N=200$ & 0.0025 & 16 & 0.038671 \\
$N=400$ & 0.0013 & 22 & 0.038673 \\
\hline
\end{tabular}

Table 4.2: Coupled sound and heat flow example: step sizes $\tau$, numbers of stages $s$ and maxima for $e$.
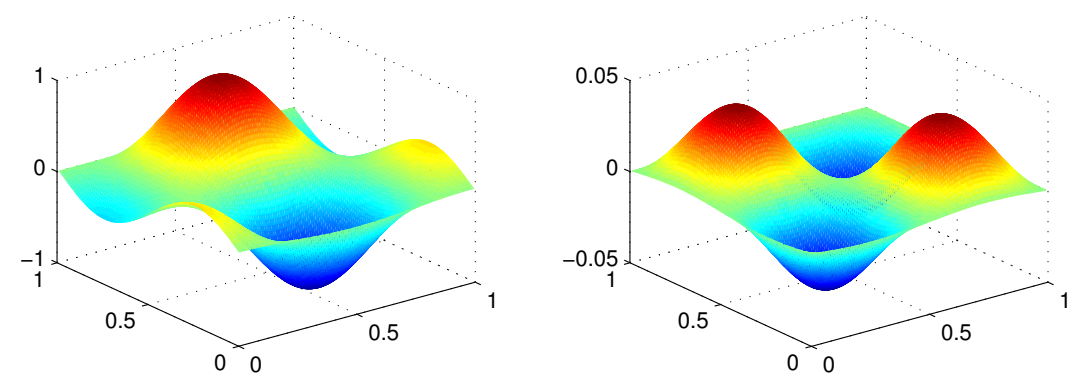

Figure 4.2: Coupled sound and heat flow example: fields computed at the $100 \times 100$ grid at time $t=1$. At the left $u_{2}$, at the right $e$.

\subsection{An advection-diffusion-reaction problem}

Our third test example is given by the 2D advection-diffusion-reaction problem (see also [16] where the same problem has been studied in $3 \mathrm{D}$ )

$$
\mathbf{u}_{t}+a_{1} \mathbf{u}_{x}+a_{2} \mathbf{u}_{y}=d \Delta \mathbf{u}+\mathbf{f}(\mathbf{u}),
$$


defined on the unit square in space and on the unit interval in time. Here $\mathbf{u}=\left(u_{1}, u_{2}\right)^{T}$, and the diffusion constant $d$ as well as the scalar velocities $a_{1}, a_{2}$ are given. The components of the nonlinear reaction term $\mathbf{f}(\mathbf{u})$ are defined by

$$
f_{1}(\mathbf{u})=-k_{2} u_{1} u_{2}+k_{1} u_{2}^{2}, \quad f_{2}(\mathbf{u})=k_{2} u_{1} u_{2}-k_{1} u_{2}^{2},
$$

where the reaction rates $k_{1}, k_{2}$ are positive constants. In this example, these constants will be given a large value causing the reactions to be stiff. As a consequence of the stiffness we will employ the IMEX version (2.5) of the RKC method.

Before continuing with the description of the advection-diffusion part in (4.11) we will first discuss the influence of the reactions on the solution. The equation $\mathbf{u}_{t}=\mathbf{f}(\mathbf{u})$ is exactly solved by

$$
u_{1}(t)=\frac{s_{0}}{k_{1}+k_{2}} \frac{k_{1}(1-\alpha)+\left(k_{1}+k_{2}\right) \alpha e^{-s_{0} k_{2} t}}{1-\alpha+\alpha e^{-s_{0} k_{2} t}}, \quad u_{2}(t)=s_{0}-u_{1}(t),
$$

where $\alpha=\left(\left(k_{1}+k_{2}\right) u_{1}(0)-s_{0} k_{1}\right) / s_{0} k_{2}$ and $s_{0}$ denotes the sum of both components which is constant in time (observe that $f_{1}(\mathbf{u})=-f_{2}(\mathbf{u})$ ). Setting $u_{1}(0)=0$ and $u_{2}(0)=s_{0}$ and choosing $k_{1}=k_{2}=k \gg 1$ we see that we have a transient phase in which both $u_{1}(t)$ and $u_{2}(t)$ rapidly converge from their initial value to $s_{0} / 2$. In the numerical experiments we set $k=10^{6}$. Combining these observations for the reaction part with the full model (4.11), we see that after the transient phase, both components are almost equal and will be advected along the characteristics of the advection operator and spatially diffused. The divergence free velocity field is defined by

$$
a_{1}(x, y)=-\sin ^{2}(\pi x) \sin (2 \pi y), \quad a_{2}(x, y)=+\sin ^{2}(\pi y) \sin (2 \pi x) .
$$

For the diffusion constant we take $d=10^{-2}$ and the initial values at $t=0$ are given by

$$
u_{1}(x, y, 0)=0, \quad u_{2}(x, y, 0)=e^{-80\left((x-0.5)^{2}+\left(y-\frac{4+\sqrt{2}}{8}\right)^{2}\right)} .
$$

These expressions will also be used to prescribe the Dirichlet boundary values for $t>0$. An accurate approximation at $t=0.5$ and $t=1$ is shown in Figure 4.3.
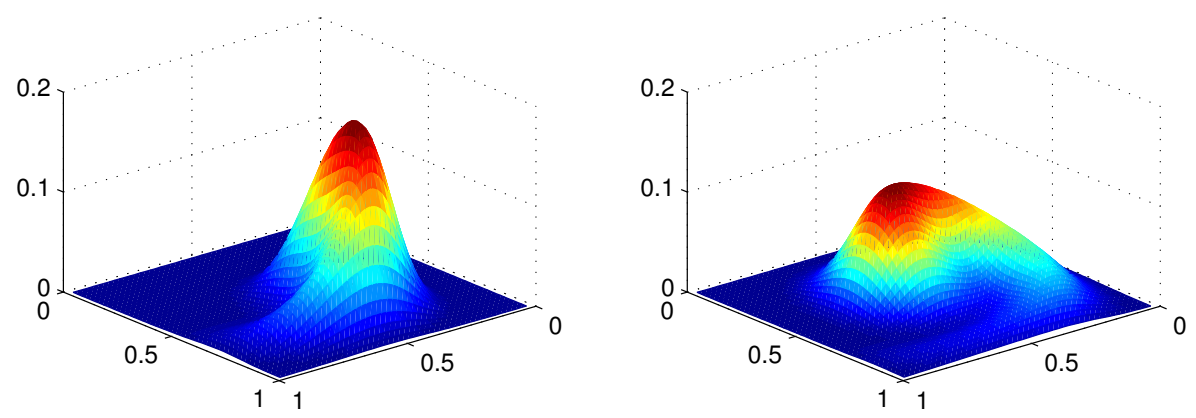

Figure 4.3: Advection-diffusion-reaction equation: a time accurate $200 \times 200$ grid solution component $u_{2}$ plotted on the $50 \times 50$ grid. At the left for $t=0.5$ and at the right for $t=1$. 
Two-step results. For the spatial discretization we use a uniform grid in both directions with mesh width $h=1 /(N+1)$, resulting in $N^{2}$ grid points. The advection operators as well as the Laplace operator are approximated by second-order symmetric differences. Because of the rapid variation of the solution in the initial part of the integration interval (caused by the stiff reaction terms), this problem will be integrated with variable step sizes. To that end, our starting point is the IMEX code IRKC from [11]. This fully automatic solver, designed for diffusion-reaction problems, needs only minor amendment: we have incorporated the two-step extension, slightly changed the local error estimator, and adapted the strategy for selecting the number of stages $s$, according to Table 3.1. Furthermore, the time step is not only determined by the local error control as in IRKC but also has to obey the CFL condition (3.7). As upper limit for $\tau$ we use $\sqrt{3} / A$ with $A=2 h^{-1}$ (cf. (4.13)).

We have integrated the above problem discretized on three spatial grids of increasing resolution, i.e., $h=1 / 100,1 / 200$, and $1 / 400$. For $T O L$-values $10^{-2}$ and $10^{-4}$ the integration results are given in Table 4.3. As is common in ODE software, TOL is a user-specified quantity to monitor (an estimate of) the local truncation error.

\begin{tabular}{ccc}
\hline Quantity & $T O L=10^{-2}$ & $T O L=10^{-4}$ \\
\hline successful steps & $146 / 245 / 476$ & $225 / 326 / 556$ \\
rejected steps & $22 / 9 / 10$ & $5 / 5 / 5$ \\
evaluations of $F_{E}$ & $851 / 1695 / 4237$ & $1136 / 2010 / 4547$ \\
evaluations of $F_{I}$ & $1857 / 3643 / 8959$ & $2732 / 4729 / 10208$ \\
maximum $s$-value used & $5 / 6 / 8$ & $5 / 6 / 8$ \\
relative error (max-norm) & $1.5_{10^{-4}} / 1.9_{10^{-5}} / 2.2_{10^{-6}}$ & $1.3_{10^{-4}} / 2.0_{10^{-5}} / 2.2_{10^{-6}}$ \\
\hline
\end{tabular}

Table 4.3: Advection-diffusion-reaction problem solved by the two-step code where the time step is prescribed by the local error control and by the CFL condition: results at $t=1$ for $h=\frac{1}{100} / \frac{1}{200} / \frac{1}{400}$.

From this table we can draw several conclusions. It turns out that in the initial, transient phase the step sizes are determined by the local error control. Once the reaction terms are in steady state, the CFL condition is more restrictive and overrules a possibly larger step size suggested by the error control. As a result, the remaining part of the integration interval is integrated with a fixed stepsize. For the various spatial grids, these CFL-based step sizes are given by $0.0086,0.0043,0.0022$, respectively. Or, equivalently, would have resulted in $115,231,462$ integration steps if all steps were chosen according to the CFL restriction. The actual number of steps taken by the solver are larger, which is caused by the requested accuracy. For both tolerance-values, this is only relevant during the transient phase. As a result of this step size selection mechanism, the global error is mainly determined by the CFLrestricted step sizes. This explaines why both $T O L$-values in Table 4.3 show approximately equal global errors if the same spatial grid is used.

The errors listed in this table are time integration errors only. That is, on each spatial grid we first determined an accurate ODE solution (using TOL $=10^{-8}$ ) with which the numerical solutions obtained with the above two TOL-values are compared. Given the fact that the solution at $t=1$ is relatively small, we decided to use the relative error, measured in the maximum norm (the errors listed in Table 4.3 correspond to the second component $u_{2}$; the errors for $u_{1}$ are almost the same). Consistent with the first conclusion we observe a smaller error on finer meshes, simply because a smaller step size was prescribed for stability reasons. After the transient phase, the solution is mainly determined by the 
linear advection-diffusion terms. This is reflected in the observed order of convergence which is close to three.

The number of $F_{E}$-evaluations equals the number of stages summed over all steps (accepted + rejected ones), augmented with inevitable evaluations needed for the overhead in an automatic solver. The number of $F_{I}$-evaluations has been averaged over the number of spatial grid points. Hence, the quotient of $F_{I}$-evaluations $/ F_{E}$-evaluations resembles the (average) number of Newton iterations that we need to solve the implicit relations. This number is seen to be in the interval $(2.1,2.4)$, which is a realistic value given the nonlinearity of the problem.

Two-step on-the-fly results. It is interesting to further examine the influence of the CFL-condition on the performance of the code. To that end, we repeated the above experiments on-the-fly. By this we mean that the instability protection for the advection terms through the CFL condition has been removed and we let the step size be determined solely on the basis of the local error control. However, the stability for the diffusion terms is still controlled by adjusting the number of stages $s$ as described in Section 3.4. For $T O L=10^{-2}$ the on-the-fly integrations failed on all three spatial grids. Apparently, the local error control was unable to prevent instability. For $T O L=10^{-4}$, however, the integrations were successful; the results are given in Table 4.4. We see that the number of steps (and consequently the global errors) are almost independent of the spatial grid. The increasing stiffness when refining the grids is only reflected in a larger number of stages. Comparing both versions of the solver, we conclude that for $T O L=10^{-4}$ the efficiencies are comparable. Still, due to its more robust behavior for a crude tolerance, the CFL-protected version is to be preferred.

\begin{tabular}{ccc}
\hline Quantity & $T O L=10^{-2}$ & $T O L=10^{-4}$ \\
\hline successful steps & unstable & $198 / 197 / 196$ \\
rejected steps & (all grids) & $5 / 7 / 7$ \\
evaluations of $F_{E}$ & & $1014 / 1414 / 2244$ \\
evaluations of $F_{I}$ & & $2435 / 3334 / 5127$ \\
maximum $s$-value used & & $7 / 12 / 25$ \\
relative error (max-norm) & & $4.7_{10^{-4}} / 4.4_{10^{-4}} / 4.3_{10^{-4}}$ \\
\hline
\end{tabular}

Table 4.4: Advection-diffusion-reaction problem solved with the on-the-fly version of the two-step code: results at $t=1$ for $h=\frac{1}{100} / \frac{1}{200} / \frac{1}{400}$.

One-step on-the-fly results. Since the diffusion coefficient $d=0.01$, all eigenvalues do have a strictly negative real part, which means that also the original one-step code described in [11] is applicable. By way of comparison we applied this code on-the-fly in the same way as the two-step code. Table 4.5 shows the results. We observe that now the crude tolerance $T O L=10^{-2}$ did not lead to instabilities. In this sense, the one-step version seems more robust for the current problem. However, although this crude tolerance leads to a rather cheap integration process (23 or 24 time steps), the relative errors are unacceptably large. Presumably there is error growth due to instability (the method has a zero imaginary stability interval and hence near the origin eigenvalues can easily jump over 
the boundary of the stability region). To obtain a more realistic accuracy, $T O L=10^{-4}$ seems more appropriate. For this tolerance we observe a similar behavior as shown in the two-step Table 4.4: the number of steps and the global errors are almost independent of the spatial grid; furthermore, the integration requires a stiffness-induced increasing number of $F$-evaluations on the finer meshes. The one-step code is slightly cheaper in number of steps due to a larger real stability interval, but given the imposed tolerance of $10^{-4}$ the temporal accuracy is too low compared to that of the two-step code. We advocate this to the higher (linear) consistency order, three versus two, and presumably there is again some error growth due to lack of stability near the imaginary axis.

\begin{tabular}{ccc}
\hline Quantity & $T O L=10^{-2}$ & $T O L=10^{-4}$ \\
\hline successful steps & $23 / 23 / 24$ & $203 / 203 / 202$ \\
rejected steps & $11 / 11 / 15$ & $6 / 6 / 7$ \\
evaluations of $F_{E}$ & $261 / 417 / 864$ & $835 / 1184 / 1888$ \\
evaluations of $F_{I}$ & $559 / 868 / 1766$ & $2031 / 2815 / 4331$ \\
maximum $s$-value used & $16 / 29 / 71$ & $6 / 10 / 21$ \\
relative error (max-norm) & $5.3_{10^{-1}} / 5.5_{10^{-1}} / 2.2_{10^{0}}$ & $1.3_{10^{-2}} / 1_{10^{-2}} / 1_{10^{-2}}$ \\
\hline
\end{tabular}

Table 4.5: Advection-diffusion-reaction problem solved with the one-step code from [11]: results at $t=1$ for $h=\frac{1}{100} / \frac{1}{200} / \frac{1}{400}$.

\section{Concluding remarks}

We have proposed the simple two-step extension (3.1) of the existing stabilized one-step methods (2.1) and (2.5). Our aim has been to create a non-zero imaginary stability interval, which we have achieved by imposing third-order consistency for linear problems. Having a non-zero imaginary stability interval allows a much wider class of problems for stabilized explicit integration. While so far the emphasis has been on advection-diffusion-reaction systems, with a non-zero imaginary stability interval different mixed PDE systems can be treated, such as damped wave equations, coupled sound and heat flow problems, Maxwell's equations taking into account thermal effects, etc.

Finally we wish to call attention to related work on stabilized explicit integration by Lebedev, Medovikov, and Abdulle, see [2, 1, 6, 7] for their methods of order two, three and four. These alternative methods are constructed in a different way, only RKC is known in a form with analytically given coefficients. A comparison between Abdulle's method and $\mathrm{RKC}$ is given in [5], Ch.V. IMEX extensions of the alternative methods are not known to the authors. A successful RKC based projection method for the incompressible Navier-Stokes equations is proposed in [20].

Acknowledgement The authors acknowledge support from the national Bsik project BRICKS. 


\section{References}

[1] A. Abdulle (2002), Fourth order Chebyshev methods with recurrence relation. SIAM J. Sci. Comput. 23, pp. 2042-2055.

[2] A. Abdulle, A.A. Medovikov (2001), Second order Chebyshev methods based on orthogonal polynomials. Numer. Math. 90, pp. 1-18.

[3] J. Douglas Jr., S. Lim, H. Lim (2003), An improved alternating-direction method for a viscous wave equation, Contemporary Mathematics 329, pp. 99-104.

[4] P.J. van der Houwen, B.P. Sommeijer (1980), On the internal stability of explicit, $m$ stage Runge-Kutta methods for large m-values. Z. Angew. Math. Mech. 60, pp. 479-485.

[5] W. Hundsdorfer, J.G. Verwer (2003), Numerical Solution of Time-Dependent Advection-Diffusion-Reaction Equations, Springer Series in Computational Mathematics, Vol. 33, Springer, Berlin.

[6] V.I. Lebedev (1994), How to solve stiff systems of differential equations by explicit methods. In: Numerical Methods and Applications. Ed. G.I. Marchuk, CRC Press, pp. $45-80$.

[7] V.I. Lebedev (2000), Explicit difference schemes for solving stiff problems with a complex or separable spectrum. Comput. Math. and Math. Phys. 40, pp. 1801-1812.

[8] H. Lim, S. Lim, J. Douglas Jr. (2006), Numerical methods for viscous and nonviscous wave equations, Appl. Numer. Math., to appear (available on line at www.sciencedirect.com).

[9] H.H. Najm, O.M. Knio (2005), Modeling low Mach number reacting flow with detailed chemistry and transport. Journal of Scientific Computing 25, pp. 263-287.

[10] R.D. Richtmyer, K.W. Morton (1967), Difference Methods for Initial-Value Problems. Second edition, John Wiley \& Sons, Interscience Publishers, New York.

[11] L.F. Shampine, B.P. Sommeijer, J.G. Verwer (2005), IRKC: An IMEX solver for stiff diffusion-reaction PDEs, CWI Report MAS-E0514, to appear in J. Comput. Appl. Math.

[12] B.P. Sommeijer, L.F. Shampine, J.G. Verwer (1997), RKC: An explicit solver for parabolic PDEs. J. Comput. Appl. Math. 88, pp. 315-326.

[13] J.G. Verwer (1996), Explicit Runge-Kutta methods for parabolic partial differential equations. Appl. Numer. Math. 22, pp. 359-379.

[14] J.G. Verwer, W.H. Hundsdorfer, B.P. Sommeijer (1990), Convergence properties of the Runge-Kutta-Chebyshev method. Numer. Math. 57, pp. 157-178.

[15] J.G. Verwer, B.P. Sommeijer (2004), An implicit-explicit Runge-Kutta-Chebyshev scheme for diffusion-reaction equations. SIAM J. Sci. Comput. 25, pp. 1824-1835.

[16] J.G. Verwer, B.P. Sommeijer, W. Hundsdorfer (2004), RKC time-stepping for advection-diffusion-reaction problems. J. Comput. Phys. 201, pp. 61-79. 
[17] P. Wesseling (1996), Von Neumann stability conditions for the convection-diffusion equation. IMA J. of Numer. Anal. 16, pp. 583-598.

[18] P. Wesseling (2001), Principles of Computational Fluid Dynamics. Springer Series in Computational Mathematics, Vol. 29, Springer, Berlin.

[19] Yin, Hong-Ming (1998), On Maxwell's equations in an electromagnetic field with the temperature effect. SIAM J. Math. Anal. 29, pp. 637-651.

[20] Z. Zheng, L. Petzold (2005), Runge-Kutta-Chebyshev projection method. Report UCSB, May 17, 2005. To appear in J. Comput. Phys. 